\title{
Diferencias de género en salud y calidad de vida en personas mayores del norte de Chile
}

\author{
Lorena Gallardo-Peralta \\ Universidad de Tarapacá, Arica, Chile. \\ Email: lgallardo@uta.cl
}

\section{Isabel Córdova Jorquera}

Universidad de Tarapacá, Arica, Chile.

Email: isabelcordova@gmail.com

\section{Marcelo Piña Morán}

Universidad Católica del Maule, Talca, Chile.

Email: pina_moran@hotmail.com

\section{Beatriz UrrutiaQuiroz}

Centro de Estudios U3E, Universidad Mayor, Santiago, Chile.

Email: beatriz.urrutia@u3e.cl

Resumen: El envejecimiento es un proceso individual y por tanto particular para cada persona que avance por sobre los 60 años, uno de los principales criterios diferenciadores del proceso de envejecimiento es el género. En esta línea, el objetivo de esta investigación es conocer las diferencias del bienestar, asociado a las condiciones de salud física -dependencia y problemas de salud-y calidad de vida, en función de la variable género. Se trata de un estudio cuantitativo y transaccional, con una muestra de 777 (488 mujeres y 289 hombres) personas mayores que residen en el extremo norte de Chile. Los resultados muestran diferencias en el proceso de envejecer entre las mujeres y los hombres. En términos objetivos los hombres envejecen mejor en su salud física y salud mental y seguridad económica y en cambio las mujeres obtienen mejores resultados en los aspectos subjetivos o de satisfacción con los diversos dominios de la calidad de vida. Las aplicaciones prácticas se discuten a partir de los resultados. calidad de vida

Palabras clave: Envejecimiento, género, problemas de salud, dependencia,

\section{Gender differences in health and quality of life among elderly people living in northern Chile}

\footnotetext{
Abstract: Aging is an individual process and therefore particular to each person over 60 years old, one of the main differentiating criteria of the aging process is gender. In this line, the objective of this research is to know the differences in well-being, associated with physical health -dependency and health issues-
} 
conditions and quality of life, depending on the gender variable. This is a quantitative and transactional study, with a sample of 777 (488 women and 289 men) elderly people that live in the North end of Chile. The results show differences in the aging process between women and men. In objective terms, men age better in their physical and mental health and economic security, whereas women obtained better results on subjective or satisfaction aspects related to the various fields of quality of life. The practical applications are discussed starting from those results.

Keywords: Aging, gender, health issues, dependence, quality of life

\section{Diferenças de gênero na saúde e qualidade de vida em idosos no norte do Chile}

Resumo: O envelhecimento é um processo individual e, portanto, particular para cada pessoa que avança além dos 60 anos, um dos principais critérios de diferenciação do processo de envelhecimento é o gênero. Nesta linha, o objetivo desta pesquisa é conhecer as diferenças de bem-estar, associadas às condições de dependência da saúde física e problemas de saúde e qualidade de vida, dependendo da variável gênero. É um estudo quantitativo e transacional, com uma amostra de 777 (488 mulheres e 289 homens) idosos que vivem no extremo norte do Chile. Os resultados diferem no processo de envelhecimento entre mulheres e homens. Em termos objetivos, os homens envelhecem melhor em sua saúde física e saúde mental e segurança econômica e, ao contrário, as mulheres obtêm melhores resultados nos aspectos subjetivos dos diferentes domínios da qualidade de vida. As aplicações práticas são discutidas a partir dos resultados. qualidade de vida

Palavras-chave: Envelhecimento, gênero, problemas de saúde, dependência,

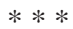

\section{Introducción}

Chile ha experimentado en los últimos años un aumento sustantivo de sus personas mayores, que se traduce en términos concretos que el 16,7\% de su población tenga más de 60 años (Encuesta de Caracterización SocioeconómicaCASEN, 2013). De esta manera, el envejecimiento de la población chilena es una realidad social que justifica estudios enfocados en este grupo de edad.

Desde diversas disciplinas, principalmente del área social, los estudios en personas mayores se han focalizado en indicadores de bienestar o calidad de vida. En otras palabras, pareciera interesar investigar cómo las personas mayores viven más años con su respectiva evaluación de "vivir mejor”.En este sentido, Urrutia, Grasso, y Guzmán (2009) refieren a la importancia de estudiar indicadores psicosociales que se centren en el vivir mejor de las personas mayores, y cómo ciertas condiciones e indicadores psicológicos y sociales influyen en el bienestar de este grupo de edad.Es importante aclararque el bienestar y la calidad de vida son constructos asociados, de carácter amplio y subjetivo sobre la valoración que hace el individuo respecto a los elementos que le satisfacen ${ }^{1}$. 
En esta línea, está bien documentada las dimensiones o variables relacionadas con una mejor vejez en los chilenos y las chilenas, como es el caso de la calidad de vida (Torres et al., 2008; Herrera, Barros y Fernández, 2011), el bienestar subjetivo (Mella et al., 2004); el bienestar psicológico (Vivaldi y Barra, 2012), la satisfacción vital (Castillo- Carniglia et al., 2012; Ramírez y Lee, 2012; Zegers, Rojas-Barahona y Förster, 2009), la salud física (Gallardo-Peralta et al. 2014), la salud mental (Gallardo-Peralta et al., 2015), la autonomía funcional (Mella et al., 2003), entre otras. Sin embargo, aún hay cierto vacío en estudios que analicen las posibles igualdades o desigualdades de género durante la vejez en nuestro país (Osorio, 2007). Al respecto, este estudio pretende ser un aporte en esta línea, analizando las posibles diferencias en las condiciones de salud (problemas de salud y nivel de independencia) y la calidad de vida. Teniendo en cuanta que el género es un factor relevante para cuantificar las necesidades de las personas mayores (Ferrer et al. 2011).

Tal como afirman Arbery Ginn(1966) género y envejecimiento están estrechamente vinculados en la vida social, esta conexión surge del cambio social propio del paso del tiempo como los acontecimientos relacionados con la edad que suceden a lo largo del tiempo. Siendo necesario aún profundizar en la comprensión de cómo se relacionan edad y género con la distribución del poder, privilegios y bienestar. Ahora bien,el análisis de las diferencias en el proceso de envejecimiento entre hombres y mujeres es complejo, pues se debe tener en cuenta, múltiples dimensiones psicosociales, especialmente las condiciones económicas y de vida (Vivaldi y Barra, 2012). En el caso de esta investigación nos centraremos como hemos adelantado en las condiciones de salud y la calidad de vida, ya que ambas dimensiones se vinculan con el bienestar en la vejez.La elección de estas variables se relaciona no solo con la importancia que han recibido en la bibliografía previa, sino también por sus características. En efecto, la calidad de vida es una medida multidimensional y subjetiva del bienestar o, dicho de otra forma, es una valoración integral del bienestar de los individuos en los dominios más significativos de la vida (Gallardo-Peralta, 2017). La salud es uno de los componentes más relevantes en al análisis de la calidad de vida global, que considera el funcionamiento - físico. psicológico y social-, el bienestar mental y social, la percepción y la satisfacción con los niveles que en estos aspectos se han alcanzado (Fernández-Mayoralas y Rojo, 2005: 43). La valoración de las condiciones de vida, tanto de la funcionalidad física (que en este estudio se analiza a través del nivel de independencia para realizar actividades de la vida diaria) y de los problemas de salud (que en esta investigación hace referencia a las patologías más recurrentes en el vejez), se tratan de dos aspectos que desencadenan una vejez con mayor o menor percepción de la calidad de vida, por tanto, una persona mayor con más dependencia y más patologías preexistentes debieran presentar una calidad de vida empeorada o menor.

\section{Salud y género en la vejez}

La salud es un aspecto íntimamente relacionado con el bienestar, por ellola OMS define la salud como "un estado de completo bienestar físico, 
mental y social y no sólo la ausencia de afecciones o enfermedades”. Aunque existen diversas maneras de conceptualizar la salud e incluso desde perspectivas sociales más holísticas, este estudio utiliza el concepto de la OMS. La salud en la vejez dependerá de las circunstancias, estilo de vida, comportamientos o acciones de las personas a lo largo de su vida (Kalache y Kickbusch, 1997). Es sabido que las mujeres enfrentan una vejez más larga, por sus altas tasas de esperanza de vida. En Chile la esperanza de vida de las mujeres de 84.49 y en los hombres es de 78.64 (mientras que para el total de la población es de 81.50). Pero el hecho de vivir más años no se relaciona, necesariamente, con la situación de vivir mejor. Prueba de ello es el hecho de que el estado de salud de las mujeres mayores es peor que el de los hombres. Las mujeres suelen presentar mayores tasas de discapacidad, más enfermedades e incluso tienen una peor salud mental (Muñoz y Espinosa, 2008).

En Chile se confirma esta asimetría en el estado de la salud, según el sexo de la persona mayor. Al respecto, los resultados del Estudio Nacional de Personas Mayores (2009) muestra que las mujeres presentan más enfermedades que los hombres, por ejemplo, para quienes reportan dos enfermedades el $23.5 \%$ son mujeres y un $19.5 \%$ son hombres. Las cifras en dependencia afirman que dos de cada tres personas mayores dependientes son mujeres. En términos específicos, la encuesta muestra que más limitaciones en las mujeres, tanto para AVD (33.1\% mujeres y un 27.3\% hombres), como para AIVD (13.8\% mujeres y 10.4 hombres). En esta misma línea, la Encuesta Nacional de Calidad de Vida (2013) informa que el 64.2\% de las mujeres tiene una percepción regular/mala de su salud (en cambio los hombres el 56.9\%), las mujeres ingieren más medicamentos (mujeres $=3.91$ y hombres $=3.08$ ) y presentan más deterioro cognitivo (mujeres 12,1\% y hombres 10,8\%).Finalmente, aunque la prevalencia de la depresiónes alta en la población de adultos mayores chilenos (Hoyl, Valenzuela y Marín, 2000), las mujeres presentan una mayor incidencia de síntomas depresivos que los hombres (von Mühlenbrock et al., 2001).

Cuando hacemos referencia a las desigualdades de género, tal como señalan Arber y Ginn (1996) tenemos que tener como referencia que el género al que se pertenece y los cambios en las relaciones entre géneros en el transcurso de la vida también influirán en nuestra manera de envejecer. En general, las desigualdades de género que afectan a las mujeres en edades avanzadas no son más que un reflejo de las que han experimentado a lo largo de vida y en especial los periodos críticos en términos fisiológicos, como la menopausia (Muñoz y Espinoza, 2008).

Hombres y mujeres han tenido distintas trayectorias de vida, distintos contextos sociales, económicos e institucionales. Mientras que las mujeres suelen tener un bajo nivel educativo, poca o a veces inexistente participación en actividades económicas formales y una vejez en mayor soledad, por la falta de pareja. En tanto los hombres, presentan una alta participación en actividades económicas, sin embargo, poca interacción con redes sociales y familiares (Salgado-de Snyder y Wong, 2007). De esta 
manera, se plantea dos grandes desigualdades de género en le vejez, por una parte, las de salud $\mathrm{y}$, por otra, las vinculadas con las condiciones de vida.

En lo que refiere a las desigualdades en salud, las mujeres suelen presentar problemas de nutrición (mal nutrición), múltiples embarazos, eventuales abortos inseguros, falta de atención en algunas enfermedades médicas, diversas manifestaciones de violencia, problemas psicológicos no tratados, trabajos en múltiples jornadas, a lo que se suma los problemas de acceso y atención en los servicios médicos (Barrantes, 2006). Pero no solo presentan un mal estado de salud física durante su trayectoria de vida, sino que además un peor estado de salud mental. Las mujeres presentan mayor riesgo o prevalencia de síntomas depresivos, aunque hay múltiples factores que pueden estar incidiendo esta situación, suelen destacar: menos oportunidades en educación, en empleo y en su desarrollo personal, la sobrecarga en el rol de cuidador sin una adecuada reciprocidad de dicho apoyo (Muñoz y Espinoza, 2008). Abundando en la línea de cuidados, el rol de “cuidador" tradicionalmente se les ha asignado a las mujeres, quienes han ejercido o bien siguen ejerciendo en edades avanzadasel rol de cuidadora, ya sea como hija, esposa, madre y/o abuela. Las mujeres, de forma especial e incluso de manera exclusiva, colaboran en el cuidado y formación de los niños y jóvenes (Piña y García, 2016). Otros factores de riesgo que afectan negativamente en la salud mental de las mujeres, están vinculados con experiencias negativas en su juventud, como puede ser la violencia de género, el trabajo no remunerado y los distintos tipos de discriminación (Barrantes, 2006).

En lo que respecta a las desigualdades en las condiciones de vida.Las mujeres suelen tener un menor nivel educativo que los hombres, dificultades en el ámbito económico, en su situación de convivencia y residencia, entre otros. Ahondando en lo económico, los ingresos de las mujeres son significativamente inferiores a los de los hombres. En lo que respecta a la convivencia y residencia, las mujeres mayores suelen vivir solas y presentar más problemas con su vivienda, en términos de equipamiento, adaptación a sus nuevas necesidades del envejecimiento, etc. (Muñoz y Espinoza, 2008).

En síntesis, las mujeres suelen acumular más riesgos a lo largo de los años vividos que desencadena en una vejez más inequitativa y compleja (Gallardo-Peralta et al., 2015, Barrantes, 2006; Acevedo y González, 2014; Salgado y Wong, 2007). A lo que agrega Castañeda (2007) la salud no puede ser tratada de la misma manera en hombres y mujeres, aun teniendo en cuenta las diferencias que se presentan a nivel biológico, ya que ambos desempeñan diferentes roles, disponen distintos espacios para llevarlos a cabo, tienen distintos estilos de vida y distintas responsabilidades. Frente a lo cual las enfermedades y exposición a riesgos serán distintas para hombres y mujeres. 


\section{Calidad de vida y género en la vejez}

Tan complejo como es la asociación entre salud y género, lo es la asociación entre calidad de vida y género. Teniendo en cuenta que la calidad de vida es un constructo multidimensional y de valoración subjetiva y objetiva. Diversos autores plantean que la calidad de vida no posee una definición unívoca (Medina y Carbonell, 2004; Rueda, 1996; Urzúa y CaqueoUrízar, 2012) al tratarse de un constructo multidimensional (Inga y Vara, 2006; Fernández- Mayoralas y Rojo, 2005). La complejidad del constructo radica no solo en su multidimensionalidad, sino que además se debe considerar el contexto en el que se investigue y las circunstancias objetivas y subjetivas del sujeto (Fernández- Ballesteros, 1997). Por esta razón nos quedaremos con la definición acuñada por Literas, Navarro y Fontanals (2010: 321) "la calidad de vida puede definirse entonces como un concepto analítico integrador, sensible a la mirada que el propio individuo tiene de sus circunstancias y entorno". En otras palabras, la calidad de vida es una evaluación que realizan las personas a partir de sus experiencias personalesen diversos aspectos que se vinculan con la satisfacción. A lo que agrega Rueda (1996) más que un acto de evaluación racional, el poder evaluar la calidad de vida supone comprender los sentimientos que experimentan los sujetos. De allí que en esta investigación abordemos la calidad de vida, en sus aspectos subjetivos y objetivos, como medida de bienestar en las personas mayores.

Otra complejidad en la valoración de la calidad de vidaes analizar o bien intentar comprender la calidad de vida en el contexto de la vejez y esto supone tener en cuenta algunos elementos distintivos de este grupo de edad. Aranibar (2001) refiere a tres ámbitos propios en la evaluación de la calidad de vida en personas mayores, la especificidad, la multidimensionalidad y los aspectos subjetivos y objetivos. La especificidad del concepto en personas mayores, en otras palabras, aunque el constructo tiene aspectos comunes con otros grupos de edad, existen aspectos y factores propios en la vejez que lo configuran. Su multidimensionalidad implica rechazar un análisis monofactorial del concepto. Por el contrario, se deben incluir factores personales y socioambientales o externos. En su valoración o evaluación se deben incluir los aspectos subjetivos y objetivos. Es decir, considerar las apreciaciones y valoraciones de los sujetos sobre los elementos de calidad de vida que disponen (más asociados a la satisfacción con los mismos), como las dimensiones objetivas de éstos (indicadores objetivos de salud, de sus relaciones sociales, etc.).

En los últimos años se han efectuado diversos estudios sobre la calidad de vida en los adultos mayores chilenos. En este sentido, las principales variables que se relacionan con calidad de vida son: disponer de redes de apoyo social, disfrutar de buena salud (funcionalidad física y autoeficacia), tener capacidad de autoaceptación y pertenecer a un entorno físico protegido. Al respecto, las redes de apoyo social, más específicamente el tener buenas relaciones sociales con la familia, amigos y comunidad, 
suele ser la variable más recurrente en la evaluación de la calidad de vida de las personas mayores chilenas. Seguido por la salud, que se operacionaliza en la capacidad de autoeficacia o independencia (capacidad funcional) y además se advierte la influencia positiva de la actividad física en esta dimensión. En esta línea, los adultos mayores experimentan diversas dolencias o enfermedades, pero destaca la "capacidad de adaptación" a sus nuevas condiciones o bien limitaciones para hacer frente a los problemas propios de la cotidianeidad. Finalmente, el pertenecer a un entorno físico protegido, ya sea la vivienda o el barrio, que se perciba como un espacio alejado de problemas sociales de violencia, drogadicción o alcoholismo (Herrera, Barros y Fernández, 2011; Osorio, Torrejón y Anigstein, 2011; Torres et al., 2008; Urzúa, et al. 2011).

No obstante, en un análisis más específico en las posibles diferencias de género y calidad de vida, se obtienen los siguientes hallazgos: ser mujer se asocia con una peor percepción de bienestar, así como una menor capacidad funcional y un menor ingreso(Herrera, Barros y Fernández, 2011) y las mujeres tienen una mayor participación social (Urzúa, et al. 2011). Pero estos estudios no se centran en analizar de manera específica las posibles diferencias de género, más bien analizan las diferencias por sexo, en la calidad de vida de las personas mayores chilenas y las condiciones de salud (independencia en AVD y problemas de salud). Igualmente,este estudio busca contribuir en la valoración de dimensiones que promueven el bienestar en edades avanzadas.

\section{Metodología}

\section{Objetivos}

General: Conocer las diferencias del bienestar, asociado a las condiciones de salud física y calidad de vida, en función de la variable género.

Específicos:

1. Evaluar las diferencias, según género, en el estado de independencia en las actividades de la vida diaria.

2. Evaluar las diferencias, según género, en los problemas de salud más recurrentes en la vejez.

3. Evaluar las diferencias, según género, en los distintos dominios de la calidad de vida.

\section{Diseño, muestra y procedimiento}

Se trata de un estudio cuantitativo y transversal. La muestra está conformada por 777 personas mayores chilenas que residen en la región de Arica y Parinacota, en el extremo norte de Chile. Para asegurar la 
representatividad, se recurrió a un muestreo estratificado por sexo, etnia y lugar de residencia (rural y urbano). Las características fundamentales de la muestra se recogen en la tabla 1 . De dichos datos conviene destacar la edad media (69.93 años; DT=7.12) y la presencia en la muestra de un porcentaje de personas mayores pertenecientes a una etnia originaria chilena (30\%), siendo la etnia Aymara la más frecuente (88\% de los casos).

Tabla 1.

Principales características de la muestra.

\begin{tabular}{|c|c|c|c|c|}
\hline \multirow[b]{2}{*}{ VARIABLES } & \multirow[b]{2}{*}{ CATEGORÍAS } & \multirow{2}{*}{$\begin{array}{c}\begin{array}{c}\text { MUJERES } \\
(\mathrm{N}=488)\end{array} \\
\mathbf{N}(\%)\end{array}$} & \multirow{2}{*}{$\begin{array}{c}\text { HOMBRES } \\
(\mathrm{N}=289) \\
\mathbf{N}(\%)\end{array}$} & \multirow{2}{*}{$\begin{array}{c}\text { TOTAL } \\
(\mathrm{N}=777)\end{array}$} \\
\hline & & & & \\
\hline & $60-69$ años & $281(58 \%)$ & $149(52 \%)$ & $430(55 \%)$ \\
\hline Edad & $70-79$ años & $153(31 \%)$ & $107(37 \%)$ & $260(34 \%)$ \\
\hline \multirow[t]{2}{*}{ (por grupos) } & 80 y más años & $54(11 \%)$ & $33(11 \%)$ & $87(11 \%)$ \\
\hline & Casado/a o con pareja & $213(44 \%)$ & $161(56 \%)$ & $374(48 \%)$ \\
\hline \multirow[t]{2}{*}{ Estado conyugal } & Soltero/a & $131(27 \%)$ & $93(32 \%)$ & $224(29 \%)$ \\
\hline & Viudo/a & $144(29 \%)$ & $35(12 \%)$ & $179(23 \%)$ \\
\hline \multirow{3}{*}{ Residencia } & Urbana: Arica & $433(89 \%)$ & $244(85 \%)$ & $677(87 \%)$ \\
\hline & Rural: Altiplano & $37(7 \%)$ & $21(7 \%)$ & $58(8 \%)$ \\
\hline & Rural:Valles & $18(4 \%)$ & $24(8 \%)$ & $42(5 \%)$ \\
\hline \multirow{4}{*}{ Escolaridad } & Sin estudios & $57(12 \%)$ & $13(5 \%)$ & $70(9 \%)$ \\
\hline & Educación básica & $194(40 \%)$ & $125(43 \%)$ & $319(41 \%)$ \\
\hline & Educación media & $188(38 \%)$ & $131(45 \%)$ & $319(41 \%)$ \\
\hline & Estudios superiores & $49(10 \%)$ & $20(7 \%)$ & $69(9 \%)$ \\
\hline \multirow[b]{2}{*}{ Trabajo } & Sigue trabajando & $194(40 \%)$ & $156(54 \%)$ & $350(45 \%)$ \\
\hline & No trabaja & $294(60 \%)$ & $133(46 \%)$ & $427(55 \%)$ \\
\hline \multirow{3}{*}{ Jefatura del hogar } & Ejerce jefatura & $212(43 \%)$ & $215(74 \%)$ & $427(55 \%)$ \\
\hline & Otra persona & $276(57 \%)$ & $74(26 \%)$ & $350(45 \%)$ \\
\hline & Pertenece a etnia originaria & $151(31 \%)$ & $81 \quad(28 \%)$ & $232(30 \%)$ \\
\hline Etnicidad & No pertenece a etnia & $337(69 \%)$ & $208(72 \%)$ & $545(70 \%)$ \\
\hline
\end{tabular}

Fuente: Elaboración propia

La aplicación del cuestionario se realizó a través de entrevista personal, obteniendo de manera previa el consentimiento informado de los participantes. Se realizó un estudio piloto con 33 personas mayores, se adaptó el lenguaje de algunas preguntas al contexto sociocultutal investigado. Los cuestionarios fueron aplicados por profesionales titulados en Trabajo Social y Psicología entre los meses de junio a agosto de 2015. La dificultad fundamental consistió en la necesidad de conseguir que la muestra contara con una población que pudiese representar la diversidad étnica y cultural (población indígena), incluyendo participantes que residieran en la zona altiplánica de los Andes. Para superar estas dificultades, se estableció contacto con las instituciones que trabajan con personas mayores (Servicio Nacional de Adultos Mayores, Municipalidad de Arica y Municipalidad de Putre), quienes facilitaron las condiciones para la aplicación de los cuestio- 
narios, aportando información sobre la composición de la población y de las principales agrupaciones de personas mayores en la región. Además, la aplicación del cuestionario en las zonas rurales y altiplánicas supuso afrontar grandes dificultades, como el difícil acceso a los poblados (por no contar con carreteras asfaltadas) o la existencia de horarios restringidos de luz pública y en los hogares de determinadas localidades (por tanto, con horarios restringidos de aplicación). En el caso específico de localidades altiplánicas se añaden las dificultades que entrevistador pueda presentar en términos físicos (por la altitud geográfica) y los rasgos culturales centrados en la desconfianza al extraño, siendo imprescindible contar con el apoyo de un agente social de la zona. Por todos esos motivos, en las zonas rurales y altiplánicas secontactaron agentes sociales clave (en clubes de personas mayores, parroquias y municipios). En resumen, el cuestionario se aplicó en cinco localidades diferentes (Arica, Putre, Socoroma, Visvirí y Codpa), asegurando de esa manera que la composición étnica y sociodemográfica de la población quedaba reflejada en la muestra. Los participantes fueron contactados a través de dos procedimientos. Cuando era posible y deseable, el primer contacto se produjo directamente por parte del equipo de investigación, que fijó una cita para realizar la entrevista. Cuando el primer contacto implicaba mayor dificultad, este se produjo a través de agentes sociales claves, como por ejemplo técnicos del ayuntamiento o los líderes vecinales más relevantes. En ambos casos, el entrevistador acudía al lugar indicado para la entrevista, cuyo tiempo de cumplimentación fue de unos 40 minutos.

\section{Instrumentos}

El cuestionario incluía antecedentes sociodemográficos básicos que se presentan en la tabla 1. De las medidas de valoración del bienestar en el envejecimiento se incluyen instrumentos de uso libre y en el caso del WHOQoL-BREF se solicitó la autorización a la OMS (World Health Organization Quality of Life Questionnaire).

Principales problemas de salud. Se utilizó el Cuestionario de Problemas de Salud de Herrera et al. (2007), es un instrumento desarrollado específicamente para la medición de las enfermedades más recurrentes en la población de adultos mayores en Chile, en el marco de la Encuesta Nacional de Calidad de Vida en la Vejez. Se trata de un inventario/checklist compuesto por 13 patologías, tales como: tensión o hipertensión, artritis/ artrosis,colesterol alto, diabetes o azúcar elevado, cataratas, osteoporosis, ataque cardiaco o problemas al corazón, enfermedad pulmonar crónica, úlcera al estómago, asma, fractura de cadera o femoral, cáncer, derrame cerebral o enfermedad vascular y enfermedad de Parkinson. El índice de consistencia interna (alphade Cronbach) del cuestionario general fue de .71.

Dependencia. Se utilizó el Índice de Barthel- Escala de actividades de la vida Diaria (AVD) de Mahoney y Barthel (1965), se trata de una escala que valora la capacidad de una persona para realizar 10 actividades básicas de la vida diaria como son comer, bañarse, vestirse, arreglarse, deposicio- 
nes, micción, ir al servicio, traslados, deambular y subir escalera. Cada actividad se le asigna una puntuación $(5,10,15)$ en función del tiempo empleado en su realización y la necesidad de ayuda para llevarlo a cabo, obteniéndose una puntuación final de 0-100. El índice de consistencia interna (alphade Cronbach) del cuestionario general fue de 90.

Calidad de Vida. Se utilizó el WHOQoL-BREF del grupo de Calidad de vida de la Organización Mundial de la Salud (1998), está conformado por un total de 26 ítems, distribuidos en 2 preguntas generalesy los 24 restantes se agrupan en los siguientes dominios: físico, psicológico, relaciones socialesy ambiente.Se trata de una valoración subjetiva que hace el sujeto de los diversos aspectos vinculados con su calidad de vida (por ejemplo, cuán satisfecho/a, conforme con ciertos recursos), pero también incluye preguntas de carácter objetivo (por ejemplo, ausencia o presencia de síntomas, dispone o no de ciertos recursos). Este cuestionario ofrece un puntaje para cada dominio y uno general, para lo cual se obtienen sumas parciales y otra general. A mayor puntaje, mayor calidad de vida. El índice de consistencia interna del cuestionario general (alphade Cronbach) fue de .89.

\section{Análisis}

Conforme a los objetivos se realiza en un primer momento un análisis descriptivo, a través de la distribución de frecuencias, media y desviación típica. En segundo lugar, para valorar las diferencias de género en las variables cualitativas se calculó la prueba $\chi^{2}$. Es decir, en aquellos casos en lo que se trataba de determinar la existencia de una diferencia entre hombres y mujeres, ya sea, en la prevalencia de alguna situación o síntoma concreto, en el nivel de independencia en las actividades de la vida diaria (categorías del Barthel) y en todos los ítems del cuestionario de calidad de vida. En tercer lugar, para la valoración de variables cuantitativas, la significación estadística de las diferencias se valoró a través de ANOVA. Los resultados se consideraron significativos cuando $\mathrm{p}<0,05$. Las variables cuantitativas analizadas fueron: sumatorio de problemas de salud, puntaje total de Barthel(nivel de independencia), dominios de calidad de vida (físico, psicológico, relaciones sociales y ambiente) y el sumatorio general de calidad de vida. El análisis de datos se realizó a través del programa SPSS, versión 23.

\section{Resultados}

En primer lugar, las tablas 2, 3 y 4 presentan los resultados obtenidos en todos y cada uno de los ítems de las tres medidas utilizadas en el estudio. Estas tablas muestran, por tanto, los resultados de manera detallada. Para cada ítem se ofrecen no solo los porcentajes, sino también el valor de $\chi^{2}$ obtenido. En la tabla 2 se ofrecen los resultados de los 13 problemas de salud más recurrentes en la vejez. Se encontraron diferencias estadísticamente significativas en dos problemas: artritis/artrosis y osteoporosis. De esta manera, las mujeres presentan más problemas de artritis/artrosis $\left(\chi^{2}=9.943 ; p=0,001\right)$ y de osteoporosis $\left(\chi^{2}=8.338 ; p=0,002\right)$ que los hombres. 


\section{Tabla 2 \\ Problemas de salud, según género}

\begin{tabular}{|c|c|c|c|}
\hline Dimensiones & $\begin{array}{c}\text { Mujeres } \\
\%\end{array}$ & $\begin{array}{c}\text { Hombres } \\
\%\end{array}$ & $\chi^{2}(p)$ \\
\hline Presión alta o hipertensión & 43.9 & 39.4 & $1.445(.229)$ \\
\hline Colesterol alto & 23.0 & 22.1 & $.067(.795)$ \\
\hline Artritis/artrosis, incluyendo osteoartritis o reumatismo & 24.0 & 14.5 & $9.943(.001)^{* *}$ \\
\hline Diabetes o azúcar elevado & 23.0 & 25.6 & $.703(.402)$ \\
\hline Osteoporosis & 10.9 & 4.8 & $8.338(.002)^{* *}$ \\
\hline Ataque cardiaco o algún otro problema al corazón & 6.4 & 8.0 & $.724(.395)$ \\
\hline Cataratas & 10.9 & 9.3 & $.453(.501)$ \\
\hline Enfermedad pulmonar crónica & 6.8 & 6.2 & $.084(.771)$ \\
\hline Úlcera de estómago o duodenal, úlcera péptica & 5.5 & 5.5 & $.000(998)$ \\
\hline Fractura de cadera o femoral & 3.9 & 4.5 & $.168(682)$ \\
\hline Derrame cerebral o enfermedad vascular cerebral & 1.2 & 1.0 & $.058(.810)$ \\
\hline Cáncer o tumor maligno & 3.5 & 2.1 & $1.252(.263)$ \\
\hline Enfermedad de Parkinson & 1.6 & 2.1 & $.196(.658)$ \\
\hline Otro(s) & 18.6 & 17.0 & $.3528 .553)$ \\
\hline
\end{tabular}

Fuente: Elaboración propia

En la tabla 3 se presentan los resultados del nivel de independencia para realizar las actividades básicas de la vida diaria. Como se puede observar en general la muestra es autovalente, si consideramos que el 77.5\% está en la categoría de independiente, un $4.9 \%$ en dependencia escasa, $14.4 \%$ en dependencia moderada, un $2.3 \%$ en dependencia severa y un $0.9 \%$ es dependiente. En el análisis del $\chi^{2}$ por las distintas categorías del Barthel no se observan diferencias estadísticamente significativas.

\section{Tabla 3 \\ Nivel de independencia, según género.}

\begin{tabular}{|c|c|c|c|}
\hline Dimensiones & $\begin{array}{c}\text { Mujeres } \\
\%\end{array}$ & $\begin{array}{c}\text { Hombres } \\
\%\end{array}$ & $\chi^{2}(p)$ \\
\hline Independiente parair al baño & 96.9 & 96.5 & $.087(.768)$ \\
\hline Independiente para lavarse la cara, las manos, peinarse, etc. & 96.5 & 95.8 & $.226(.635)$ \\
\hline Independiente para vestirse & 93.4 & 93.8 & $.081(.960)$ \\
\hline Independiente para comer & 95.1 & 94.1 & $.648(.723)$ \\
\hline Independiente ir al WC, quitarse y ponerse la ropa & 94.5 & 90.7 & $4.196(.123)$ \\
\hline Independiente para ir del sillón a la cama & 85.2 & 85.1 & $2.521(.471)$ \\
\hline Independiente para caminar ( 50 metros) & 84.4 & 84.8 & $.428(.934)$ \\
\hline Independiente para subiry bajar escaleras & 80.9 & 81.7 & $.078(.962)$ \\
\hline Continente para orinar & 95.5 & 95.2 & $.090(.956)$ \\
\hline Continente para deposiciones & 97.5 & 98.3 & $1.290(.525)$ \\
\hline
\end{tabular}

Fuente: Elaboración propia

Finalmente, en la tabla 4 se muestran los resultados obtenidos en los distintos ítems y dimensiones de calidad de vida. A través de la prueba del $\chi^{2}$ se valoran todos los ítems del cuestionario en función de posibles diferencias según género. Los resultados muestran diferencias estadísticamente 
significativas entre hombres y mujeres. Los ítems que muestran una ventaja o mejores resultados en la calidad de vida de las mujeres son: mayor satisfacción general con la calidad de vida $\left(\chi^{2}=18.389 ; p=0,001\right)$; mayor satisfacción consigo mismas $\left(\chi^{2}=21.023\right.$; $\left.p=0,000\right)$; mayor satisfacción con sus vínculos o relaciones sociales $\left(\chi^{2}=11.783 ; p=0,01\right)$, mayor satisfacción con las condiciones del lugar en que residen o vive $\left(?^{2}=14.530 ; p=0,006\right)$ y mayor satisfacción con los servicios de salud $\left(\chi^{2}=12.712 ; p=0,01\right)$. En contraposición los hombres muestran mejores resultados en los siguientes ítems: presentan menos síntomas depresivos $\left(\chi^{2}=14.042 ; p=0,007\right)$ y disponen del dinero para satisfacer sus necesidades $\left(\chi^{2}=16.022 ; p=0,003\right)$.

\section{Tabla 4
Calidad de vida, según género.}

\begin{tabular}{|c|c|c|c|}
\hline Dimensiones & $\begin{array}{c}\text { Mujeres } \\
\%\end{array}$ & $\begin{array}{c}\text { Hombres } \\
\%\end{array}$ & $\chi^{2}(p)$ \\
\hline \multicolumn{4}{|l|}{ PREGUNTAS GENERALES } \\
\hline Calificaria como buena muy buena su calidad de vida & 55.3 & 48.1 & $18.0389(.001)^{* *}$ \\
\hline Se siente satisfecho con estado de salud & 38.5 & 37 & $8.172(.085)$ \\
\hline \multicolumn{4}{|l|}{ DOMINIO 1: FISICO } \\
\hline El dolor fisico impide hacer lo que necesita & 14.3 & 12.8 & $3.593(.464)$ \\
\hline Necesita de tratamiento médico para funcionar & 21.5 & 18.4 & $8.117(.087)$ \\
\hline Tiene suficiente energia & 59.6 & 62.6 & $6.189(.185)$ \\
\hline Se puede desplazar de un lugar a otro & 76.6 & 78.5 & $1.211(.876)$ \\
\hline Se siente satisfecho con su sueño & 35.7 & 33.2 & $3.353(.501)$ \\
\hline Se siente satisfecho con su capacidad para hacer actividades cotidianas & 50 & 47 & $3.313(.507)$ \\
\hline Se siente satisfecho con su capacidad de trabajo & 43 & 42.5 & $4.312(.365)$ \\
\hline \multicolumn{4}{|l|}{ DOMINIO 2: PSICOLOGICO } \\
\hline Disfruta la vida & 57.5 & 53.2 & $3.884(.422)$ \\
\hline Siente que la vida tiene sentido & 64.3 & 59.9 & $3.763(.439)$ \\
\hline Es capaz de concentrarse & 43.9 & 48.1 & $3.095(.542)$ \\
\hline Acepta apariencia fisica & 79.7 & 83.1 & $3.887(.422)$ \\
\hline Se siente satisfecho consigo mismo & 60.8 & 54.3 & $21.023(.000)^{* * *}$ \\
\hline Experimentan sentimientos de tristeza... depresión & 10.7 & 6.6 & $14.042(.007)^{* *}$ \\
\hline \multicolumn{4}{|l|}{ DOMINIO 3: RELACIONES SOCIALES } \\
\hline Se siente satisfecho con sus relaciones personales & 59.6 & 57.5 & $11.783(.019)^{*}$ \\
\hline Se siente satisfecho con su vida sexual & 18.1 & 25.8 & $8.351(.080)$ \\
\hline Se siente satisfecho con el apoyo de amigos & 42.2 & 39.7 & $7.823(.098)$ \\
\hline \multicolumn{4}{|l|}{ DOMINIO 4: AMBIENTE } \\
\hline Se siente seguro en su vida diaria & 53.7 & 50.5 & $3.627(.459)$ \\
\hline Cuán saludable es su ambiente fisico & 59.4 & 59.9 & $4.098(.393)$ \\
\hline Dispone de dinero para cubrir necesidades & 26.3 & 28.1 & $16.022(.003)^{* *}$ \\
\hline Dispone de información & 52.4 & 49.8 & $5.993(.200)$ \\
\hline Puede realizar actividades de recreación & 33.8 & 30.8 & $7.998(.092)$ \\
\hline Se siente satisfecho con las condiciones de lugar que vive & 63.9 & 55.8 & $14.530(.006)^{* *}$ \\
\hline Se siente satisfecho con los servicios de salud & 56.4 & 53.6 & $12.712(.013)^{*}$ \\
\hline Se siente satisfecho con la movilización del barrio & 52.9 & 51.6 & $3.137(.535)$ \\
\hline
\end{tabular}
${ }^{*} p<.05,{ }^{* *} p<.01,{ }^{* * *} p<.001$

Fuente: Elaboración propia

En la tabla 5 se muestran los resultados en las medidas utilizadas. En este caso, se realiza un análisis de las diferencias estadísticamente significativas en los sumatorios de cada dimensión o escala. Dicho de otra forma, no se toman los ítems uno a uno, sino que se ofrecen los resultados para las dimensiones definidas por varios ítems. Los resultados muestran diferencias entre hombres y mujeres. Sin embargo, cuando se analiza la sumatoria de problemas de salud y el puntaje total de independencia, no se observan 
diferencias estadísticamente significativas según el género. Pero sí hay diferencias en análisis de los cuatro dominios y en el puntaje total de la calidad de vida, en todos ellos la $p=0,000$. Los datos son positivos en los hombres en los dominios: físico y psicológico. En cambio, los resultados son favorables para las mujeres en los dominios: relaciones sociales y ambientes. En tanto, en la valoración general de calidad de vida (esto se refiere al puntaje total del WHOQoL-BREF), las mujeres muestran mejores resultados que los hombres.

Tabla 5

Diferencias de género en problemas de salud, nivel de independencia y calidad de vida

\begin{tabular}{lcccc}
\hline & MUJERES & HOMBRES & F & $\mathrm{p}$ \\
& $M(D E)$ & $M(D E)$ & & \\
\hline Problemas de salud & $1.83(1.3)$ & $1.62(1.3)$ & 1.38 & .191 \\
Nivel de independencia & $95.31(17.89)$ & $94.60(14.65)$ & 1.252 & .222 \\
Dominio físico & $101.11(21.00)$ & $101.76(21.74)$ & 12.10 & $.000^{* * *}$ \\
Dominio psicológico & $91.07(15.40)$ & $91.18(16.90)$ & 5.60 & $.000^{* * *}$ \\
Dominio relaciones sociales & $39.39(9.58)$ & $39.25(11.41)$ & 3.05 & $.000^{* * *}$ \\
Dominio ambiente & $113.25(22.20)$ & $110.38(23.55)$ & 3.67 & $.000^{* * *}$ \\
Sumatorio Calidad de vida & $35.85(3.67)$ & $35.73(4.10)$ & 7.93 & $.000^{* * *}$ \\
\hline
\end{tabular}

Fuente: Elaboración propia

\section{Discusión y conclusiones}

Los resultados de este estudio confirman la heterogeneidad del proceso de envejecimiento según el sexo, frente a lo cual se confirma la premisa de que las condiciones de riesgo en la salud y en la calidad de vida están vinculadas a las trayectorias de vida diferentes para las mujeres y los hombres. Pero quizás la mayor aportación de esta investigación es en la línea de identificar los factores protectores o de riesgo de cara a promover el bienestar en la vejez en las chilenas y los chilenos.

En el ámbito físico se observa que las mujeres padecen más problemas de salud o enfermedades crónicas no transmisibles como artritis/artrosis y osteoporosis. Ambos problemas de salud son de carácterosteroarticulares y suelen ser muy comunes en adultos mayores chilenos (Fernández, Herrera y Valenzuela, 2014; Tapia et al., 2010). Enfermedades reumáticas como la artritis/artrosis producen muchas molestias y limitaciones funcionales en la vejez, además de ser una de las patologías más diagnosticadas en adultos mayores (Tamayo y Rebolledo, 2010) y con mayor prevalencia en mujeres (Castillo- Carniglia et al., 2012) en Chile. En el caso de la osteoporosis, la prevalencia también suele ser mayor en las mujeres (postmenopáusicas) y está relacionada con la inadecuada ingesta de calcio (Gajardo, 2000). Estas enfermedades reumáticas se encuentran entre las causas más frecuentes de morbilidad, incapacidad y utilización de servicios de saluden nuestro 
país(Tamayo y Rebolledo, 2011). Que las mujeres padezcan más estas enfermedades reumáticas viene a ratificar su mayor exposición, a lo largo de la vida, a factores de riesgo en salud., incluso pasados los 65 años las mujeres chilenas con osteoporosis muestran una inadecuada ingesta de calcio (Gajardo, 2000).Teniendo en cuenta que estas enfermedades están relacionadas con el estilo de vida, nutrición (ingesta inapropiada de calcio y/o obesidad) y hacer ejercicios, es posible promocionar o prevenir la prevalencia o bien la gravedad de estas patologías.

En el ámbito de la dependencia, no se observa diferencias estadísticamente significativas entre hombres y mujeres. Aunque la evidencia empírica nacional (Herrera, Barros y Fernández, 2011; SENAMA, 2009) e internacional (Ferrer et al., 2011; Muñoz y Espinoza, 2008) confirma que las mujeres en la vejez presentan más dependencia. Como ya adelantábamos una posible explicación a esto se debe a las características de la muestra., tratándose de una muestra relativamente joven (55\% tiene menos de 70 años) y la baja incidencia de la dependencia, solo un $0.9 \%$.

En los dominios analizados de la calidad de vida, los resultados son distintos para hombres y mujeres. En lo que respecta al dominio físico los resultados son más positivos para los hombres, cuya valoración general en esta dimensión es más alta que en las mujeres. Este dato es concordante con los obtenidos en el estudio efectuado por Castillo- Carniglia et al. (2012), quienes observan que los hombres informan de una buena salud (autoreporte que califica la salud como buena, muy buena y excelente). Como hemos analizado también puede influir negativamente en las condiciones de salud de las mujeres el padecer más enfermedades crónicas.

En tanto el dominio psicológico los datos son dispares, no obstante, en términos generales favorables para los hombres. Por una parte, las mujeres se muestran más satisfechas consigo mismas, lo que debiera redundar en una mejor calidad de vida en este dominio, teniendo en cuenta que autoaceptación se vincula con mayor bienestar en la vejez. Al respecto, Urzúa et al. (2011) afirman que los adultos mayores que desarrollan la autoaceptación debieran mejorar su capacidad para solucionar problemas, estarían más satisfechos con las actividades que realizan y se podrían relacionar de manera íntima con otros. Sin embargo, los resultados muestran que los hombres obtienen una mejor valoración general del dominio psicológico y además tienen menos sintomatología depresiva. Aunque este dominio valora otros aspectos del bienestar psicológico (satisfacción vital, autoaceptación, propósito con la vida, entre otros) la mayor prevalencia de síntomas o sentimiento negativos (tristeza, desesperanza, ansiedad, depresión) en las mujeres mayores de 60 años, viene a confirmar una situación recurrente de riesgo asociadas al género. En otras palabras, "ser mujer continúa siendo un factor de riesgo para la depresión en la vejez” (Riquelme et al., 2008: 6-7).

En el dominio relaciones sociales las mujeres obtienen resultados positivos. Al respecto, las mujeres obtienen una valoración general positi- 
va en esta dimensión y en términos específicos, se muestran más satisfechas con sus vínculos sociales. En esta misma línea, Castillo- Cariglia et al. (2012) confirman más apoyo social en las mujeres mayores chilenas. Lo que es concordante con la evidencia empírica internacional, que confirma que las mujeres mayores poseen fuentes de apoyo social más diversas, más amplias, son mayores proveedoras de apoyo, perciben y están más satisfechas con el apoyo social (Antonucci, 1985; Gurung, Taylor y Seeman, 2003; Okamoto y Tanaka, 2004).

En el dominio ambiente las mujeres obtienen mejores resultados, aunque se aprecia un dato que es contradictorio. Como señalábamos en la valoración general del ambiente las mujeres tienen resultados mejores y en términos concretos, están más satisfechas con las condiciones del hogar y con el acceso a los servicios médicos. Pero lo que es incongruente es el hecho de que los hombres dispongan de los recursos económicos suficientes para satisfacer sus necesidades y no así las mujeres, aun así, muestren mejores resultados en este dominio. Estudios nacionales confirman esta situación desigual (Castillo- Cariglia et al., 2012; Herrera, Barros y Fernández, 2011), es decir, que los hombres dispongan de seguridad económica, en cambio las mujeres estén en una situación de precariedad en lo económico. Esta incongruencia en la valoración positiva del ambiente en las mujeres, aun no teniendo los recursos económicos para satisfacer las necesidades básicas, puede tener una explicación en el hecho de que ellas han experimentado en sus trayectorias de vida peores condiciones económicas que los hombres, por tanto,pueden estar "adaptadas”, “resignadas” o "habituadas” a esta situación desigual.

Por último, las mujeres presentan una valoración más alta en la satisfacción general con la calidad de vida, es decir, en el puntaje total del cuestionario aplicado. Aquí retomamos la noción general de la calidad de vida, como un constructor subjetivo, individual, dinámico y por sobretodo basado en la percepción de los sujetos (Fernández- Mayoralas et al., 2005). Este estudio muestra las contradicciones en la percepción de la calidad de vida, es decir, las mujeres presentan peores indicadores en el dominio físico y psicológico (síntomas depresivos) y dificultades en la satisfacción de necesidades (recursos/seguridad económica), no obstante realizan una valoración general más alta que los hombres en su calidad de vida. Lo que podría encontrar una respuesta en la dominada "paradoja del bienestar", pese a las dificultades del envejecimiento (tales como: deterioro físico, muerte de los viejos amigos y familiares), las personas mayores no informan una disminución concomitante en la mayoría de los aspectos del bienestar (Inga y Vara, 2006). Más bien lo contrario, frente a situaciones adversas, las personas mayores reportan altos sentimientos de bienestar y de satisfacción general.

Este estudio tiene aplicaciones prácticas desde el punto de vista de la gerontología social. En primer lugar se debe considerar que la gerontología como ciencia no solo abarca el estudio y trabajo en torno a la etapa de vejez, sino que aborda todo el proceso de envejecimiento, entendido éste 
como el proceso que vive cada ser humano a lo largo de su existencia desde la gestación hasta la muerte (Orellana, 2011). De este modo, contemplando que los seres humanos envejecemos a lo largo de todo el ciclo vital y no solo una vez que cumplimos determinada edad (60 años en Chile), se hace necesaria la educación gerontológica antes de entrar a la etapa de vejez, para que se llegue en las mejores condiciones posibles, prolongando el máximo de tiempo la autonomía, independencia y calidad de vida. Lo anterior, a través de actividades formativas en diversas áreas, por ejemplo, en estilos de vida saludable, (nutrición/alimentación, actividad física, cuidado del sueño, uso y abuso de sustancias nocivas, entre otras); prevención de enfermedades crónicas, especialmente aquellas que afectan a las mujeres en la postmenopausia, pero que son necesarias de prevenir con antelación, por ejemplo, la ingesta de calcio debe ser apropiada las tres primeras décadas de vida para obtener una adecuada mineralización ósea la que alcanza su pico a los 30 años y luego va en descenso (Marín, 2005); desarrollo de redes sociales y de apoyo post jubilación, principalmente dirigido a los hombres, para quienes, en muchos casos, dejar de trabajar es sinónimo de pasividad, lo que los excluye de la mayoría de las formas de interacción y sociabilidad (Orellana y Ríos, 2010).

Del estudio también se desprende la idea de que los profesionales de la gerontología no podemos tratar al grupo de mayores como un colectivo homogéneo, se reafirma la impresión de que las personas en la medida que envejecen se van haciendo diferentes unas de otras, esta distinción en el envejecimiento depende de diversos factores, además del género: la edad, antecedentes étnicos y culturales, zonas de residencia rural o urbana, si viven solos o con familia, su condición socioeconómica, nivel educativo, entre otros (Forttes y Urrutia, 2010). De este modo cobra relevancia el pensar el trabajo gerontológico desde los enfoques de Curso de Vida (Blanco, 2001) y de Atención Gerontológica Centrada en la Persona (Martínez, 2011), que tienen en su base el paradigma de la trayectoria de vida de los sujetos, considerando relevante su biografía al momento de proyectar un plan de intervención, contemplando de esta forma las particularidades de los mayores.

Por otra parte, en gerontología social es clave trabajar desde los aspectos positivos y no solo desde las pérdidas que ocurren en la vejez, por lo tanto, el estudio al identificar en cuales aspectos se encuentran más satisfechos hombres y mujeres en relación con su percepción de salud y calidad de vida, aporta en la identificación de distintas fortalezas en ambos géneros desde las cuales se puede trabajar de manera diferenciada para así desarrollar al máximo el potencial de las personas mayores.

Finalmente, el estudio muestra también la satisfacción con las redes personales, en este sentido no podemos olvidar al intervenir con mayores que asimismo, es necesario trabajar con su entorno social, familia y amigos para de este modo poder cambiar hábitos y estilos de vida en post de mejorar su salud y condiciones de vida. 
A modo de conclusión, esta investigación confirma la complejidad en la valoración del bienestar - centrado en la calidad de vida y en las condiciones de salud- en las personas mayores. No solo cuando el análisis discrimina por género, es decir, considerando las posibles diferencias entre mujeres y hombres. Además, la complejidad estará dada por la valoración objetiva o subjetiva del bienestar. Los aspectos objetivos, son favorables en los hombres, quienes padecen menos enfermedades crónicas (muchas de ellas invalidantes), experimentan menos sentimientos negativos o depresivos y dicen tener el dinero suficiente para cubrir sus necesidades (seguridad económica). En contraste, los aspectos subjetivos son favorables en las mujeres, quienes están satisfechas consigo mismas, están más satisfechas con sus redes sociales, están más satisfechas con las condiciones del hogar y están más satisfechas con los servicios de salud. Por tanto, es necesario seguir profundizando el análisis sobre los aspectos subjetivos versus los objetivos en la valoración del bienestar en la vejez.

Esta investigación presenta limitaciones. Por una parte, se trata de un diseño de investigación de tipo transversal y no longitudinal, lo que lleva a tener cuidado o bien cautela en la interpretación de dirección causal de los resultados obtenidos. Una de las limitaciones teóricas de este estudio es analizar la salud desde una mirada individual, por tanto, desconociendo los aspectos colectivos, políticos, económicos y culturales de este constructo, lo que sin duda dificulta el análisis integrador de la calidad de vida. Otra limitación se vincula con las características de la muestra, como hemos visto se trata de una muestra joven dentro del grupo de adultos mayores (la mayoría tiene menos de 70 años) y con una baja incidencia de dependencia, de esta manera, sería interesante en futuras investigaciones analizar las diferencias de género en población mayor de 80 años y con un deterioro físico y cognitivoOtras líneas de investigación que abre este estudio son ahondar en la incidencia de la etnicidad en la valoración del bienestar y especialmente de la calidad de vida en personas mayores chilenas, esto puede ser un aporte especialmente enriquecedor a la diversidad étnica de nuestro país en edades avanzadas. Junto con las limitaciones planteadas, los autores estiman la conveniencia de realizar estudios cualitativos que puedan dar sentido las contradicciones del bienestar entre hombres y mujeres, como hemos visto este estudio deja en evidencia ciertas paradojas subjetivas y objetivas en la valoración de la salud y la calidad de vida que pudiesen ser valorados desde la perspectiva subjetiva y cualitativa de las personas mayores.

\section{Agradecimientos}

Esta investigación ha sido financiada por el Fondo Nacional de Desarrollo Científico y Tecnológico (FONDECYT Nº 11140020) del Gobierno de Chile y el Proyecto de Investigación de Pregrado de la Universidad de Tarapacá (3761-16). 
Polis, Revista Latinoamericana, $N^{\circ}$ 49, 2018

\section{Nota}

${ }^{1}$ Véase en mayor profundidad en Fernández- Mayoralas et al., 2003. 


\section{Bibliografía}

Acevedo, J. y González, J. (2014). No envejecemos igual: la religiosidad y el género en adultos mayores del noreste de México. Reflexiones, 93(1), 133-144.

Antonucci, T.C. (1985). Personal characteristics, social support, and social behavior. En R.H. Binstock y E. Shanas (Eds.), Handbook of aging and the social sciences (pp. 94-128). Nueva York, EstadosUnidos: Van Nostrand Reinhold.

Aranibar, P. (2001). Acercamiento conceptual a la situación del adulto mayor en América Latina. Santiago, Chile: CEPAL

Arber, S. y Ginn, J. (1996). Relación entre género y envejecimiento. Enfoque sociológico. Madrid: NARCEA.

Barrantes, M. (2006). Género, vejez y salud. Acta Bioética, 12(2), 193-197.

Blanco, M. (2011). El enfoque del curso de vida: orígenes y desarrollo. Revista Latinoamericana de Población, 5 (8), 5-31.

Castañeda, I.E. (2007). Reflexiones teóricas sobre las diferencias en salud atribuibles al género. Revista Cubana de Salud Pública, 33(2), 1-20.

Castillo-Carniglia, A., Albala, C., Gangour, A.D. y Uauy, R. (2012). Factores asociados a satisfacción vital en una cohorte de adultos mayores de Santiago, Chile. Gaceta Sanitaria, 26(5), 414-20.

Fernández- Ballesteros, R. (1997). Calidad de vida en la vejez: condiciones diferenciales. Anuario de Psicología, 73, 89-104.

Fernández- Mayoralas, G. y Rojo, F. (2005). Calidad de vida y salud: planteamientos conceptuales y métodos de investigación. Territoris, 5, 117-136.

Fernández- Mayoralas, G., Rojo-Pérez, F., Abellán, A. y RodríguezRodríguez, V. (2003). Envejecimiento y salud. Diez años de investigación en el CSIC. Revista Multidisciplinar de Gerontología, 13(1), 43-46.

Fernández, B., Herrera, S. y Valenzuela, E. (2014). Tercera encuesta nacional Calidad de Vida en la Vejez. Santiago, Chile: Pontificia Universidad Católica de Chile

Ferrer, A., Badía, T., Formiga, F., Almeda, J. Fernández, C. y Pujol, R. (2011). Diferencias de género en el perfil de salud de una cohorte de 85 años. Estudio Octabaix. Atención Primaria, 43(11), 577-584. 
Forttes, A. y Urrutia, B. (2010). Imágenes culturales de la vejez. En Preparación a los cambios de la madurez vital y laboral, Santiago, Chile: s.n: $40-49$.

Gajardo, H. (2000). Situación de la osteoporosis en Chile. Revista Médica de Chile, 128(7), 809-812.

Gallardo-Peralta, L. (2017). The relationship between religiosity/spirituality, social support, and quality of life among elderly Chilean people. International Social Work, 60, 1498-1511.

Gallardo-Peralta, L. P., Barrón López-de-Roda, A., Sánchez-Moreno, E., \& Arias-Astray, A. (2014). Dimensiones estructurales y funcionales del apoyo social y salud en personas mayores en Chile. Revista Mexicana de Psicología, 31(2), 187-197.

Gallardo-Peralta, L.P., Sánchez-Moreno, E., Barrón, A., Astray, A. (2015). Ethnicity, social support, and depression among elderly Chilean people. The Journal of Psychology, 149(6), 601-629.

Gurung, R. A., Taylor, S. E. y Seeman, T. E. (2003). Accounting for changes in social support among married older adults: Insights from the MacArthur studies of successful aging. Psychology and Aging, 18(3), 487-496.

Herrera, M.S., Barros, C. y Fernández, M.B. (2011). Predictors of Quality of Life in Old Age: A Multivariate Study in Chile. Journal of Population Ageing, 4, 121-139.

Herrera, S., Barros, C. y Fernández, B. (2007). Primera encuesta nacional Calidad de Vida en la Vejez. Santiago, Chile: Pontificia Universidad Católica de Chile.

Hoyl,T., Valenzuela, E. y Marín, P.P. (2000). Depresión en el adulto mayor: evaluación preliminar de la efectividad, como instrumento de tamizaje, de la versión de 5 ítems de la Escala de Depresión Geriátrica. RevistaMédica de Chile, 128(11), 1199-1204.

Inga, J. y Vara, A. (2006). Factors associated to life satisfaction in adults over 60 years old in Lima-Peru. UniversitasPsychologica, 5(3), 475486.

Kalache, A. y Kickbusch, I. (1997). A global strategy for healthy ageing. World Health, 50(4), 4-5.

Literas, L., Navarro, A., y Fontanals, M.D. (2010). Diseño y validación de una escala de de satisfacción y calidad de vida para usuarios de centros residenciales y sociosanitarios. Revista Española de Geriatría y Gerontología, 45(6), 320-325. 
Mahoney, F.I. y Barthel, D.W. (1965). Functional evaluation: the Barthel Index. Maryland state Medical Journal, 14, 61-65.

Marín, P. y Gac, H. (2005). Manual de Geriatría y Gerontología. Santiago, Chile: Ediciones Universidad Católica de Chile.

Martiìnez, T. (2011). La atención gerontológica centrada en la persona. $1^{\text {a }}$ Edición. Vitoria-Gasteiz: EuskoJaurlaritzaren Argitalpen Zerbitzu Nagusia: Servicio Central de Publicaciones del Gobierno Vasco, 13- 105.

Medina, M.E. y Carbonell, M.C. (2004). Evaluación de la calidad de vida de las personas mayores en el Municipio de Murcia. Portularia, 4, 179186.

Mella, R., Alvear, M., Carrillo, B. y Caire, V. (2003). Valoración de las funciones mentales y la comunicación en adultos mayores mapuches y no mapuches en áreas rurales del sur de Chile. Revista Médica de Chile, 131, 1257-1265.

Mella, R., González, L., D’appolonio, J., Maldonado, I., Fuenzalida, A. y Díaz, A. (2004). Factores asociados al bienestar subjetivo en el adulto mayor. Psykhe, 13, 79-89.

Ministerio de Desarrollo Social (2013). Encuesta de caracterización socioeconómica nacional (CASEN) 2013: Síntesis de resultados de adultos mayores. Chile.

Muñoz, F. y Espinosa, J.M. (2008). Envejecimiento activo y desigualdades de género. Atenciónprimaria, 40(6), 305-309.

Okamoto, K. y Tanaka, Y. (2004). Gender differences in the relationship between social support and subjective health among elderly persons in Japan. Preventive Medicine, 38(3), 318-322.

Orellana, V. (2011). Introducción a la gerontología social y aspectos básicos de geriatría. En Gerontología Social para Encargados Municipales del Adulto Mayor. Santiago, Chile: Pontificia Universidad Católica de Chile.

Orellana, V. y Ríos, R. (2010). Preparación a los cambios de la madurez vital y laboral. Santiago, Chile: Pontificia Universidad Católica de Chile.

Osorio, P. (2007). Construcción Social de la Vejez y Expectativas ante la Jubilación en Mujeres Chilenas. Universum (Talca), 22(2), 194-212.

Osorio, P., Torrejón, M.J. y Anigstein, M.S. (2011). Calidad de vida en personas mayores en Chile. Revista Mad-Universidad de Chile, 24, 61-75. 
Piña, M. y García, L. (2016). Socio-Cultural Connections and Ruptures: Social Roles of Older Adults in Chile. Journal of Population Ageing, 9(3), 263-280.

Ramírez, M. y Lee, S.L. (2012). Factores asociados a la satisfacción vital en adultos mayores de 60 años. Polis (Santiago), 11(33), 407-428.

Riquelme, A., Ortigosa, J.M., Martín, M. y Lechuga, A. (2008). Depresión y envejecimiento: algunas cuestiones de interés respecto a la investigación epidemiológica. Revista Multidisciplinar de Gerontología, 18(2), 76-80.

Rueda, S. (1996). Habitabilidad y calidad de vida. Cuadernos de Investigación Urbanística, 42, 29-34.

Salgado-de Snyder, N. y Wong, R. (2007). Género y pobreza: determinantes de la salud en la vejez. Salud Pública de México, 49(4), 515-521.

Servicio Nacional de Adultos Mayores (2010). Estudio Nacional de la Dependencia en las Personas Mayores. Santiago, Chile: Gráfica Puerto Madero.

Tamayo, M. y Rebolledo, J. (2011). Garantía de oportunidad, artrosis y autorreporte de salud en personas con artrosis en una comuna de la Región Metropolitana. Revista Médica de Chile, 139(12), 1617 1623.

Tapia, C., Varela, H., Barra, L., Ubilla, M.D., Iturra, V., Collao, C.y Silva, R. (2010). Valoración multidimensional del envejecimiento en la ciudad de Antofagasta. Revista Médica de Chile, 138(4), 444-451.

Torres, M., Quezada, M., Rioseco, R. y Ducci, M.E. (2008). Calidad de vida de adultos mayores pobres de viviendas básicas: Estudio comparativo mediante uso de WHOQol-BREF. Revista Médica de Chile, 136, 325-333.

Urrutia, A.I., Grasso, L.T.P., y Guzmán, E.R. (2009). Construcción y validación de un instrumento multivariable para la evaluación de calidad de vida en ancianos. Revista Evaluar, 9, 54-71.

Urzúa, A. y Caqueo-Urízar, A. (2012). Calidad de vida: una revisión teórica del concepto. Terapia Psicológica, 30 (1), 61-71.

Urzúa, A., Bravo, M., Ogalde, M. y Vargas, C. (2011). Factores vinculados a la calidad de vida en la adultez mayor. Revista Médica de Chile, 139(8), 1006-1014.

Vivaldi, F. y Barra, E. (2012). Bienestar psicológico, apoyo social percibido 
y percepción de salud en adultos mayores. Terapia psicológica, 30(2), 23-29.

VonMühlenbrock, F., Gómez, R., González, M., Rojas, A., Vargas, L. y von Mühlenbrock, C. (2011). Prevalencia de Depresión en pacientes mayores de 60 años hospitalizados en el Servicio de Medicina Interna del Hospital Militar de Santiago. RevistaChilena de Neuro-psiquiatría, 49(4), 331-337.

WHOQOL Group. (1998). The World Health Organization quality of life assessment (WHOQOL): development and general psychometric properties, Social Science \& Medicine, 46(12), 1569-1585.

Zegers, B., Rojas-Barahona, C. y Förster, C. (2009). Validez y confiabilidad del índice de satisfacción vital (LSI-A) de Neugarten, Havighurst\&Tobin en una muestra de adultos y adultos mayores en Chile. Terapia psicológica, 27, 15-26.

Recibido: 01.01.17

Aceptado: 31.01 .18 